Schmerz 2012 $26: 644-646$

DOI 10.1007/s00482-012-1267-9

Online publiziert: 26 . November 2012

(c) Deutsche Schmerzgesellschaft e.V.

Published by Springer-Verlag Berlin Heidelberg -

all rights reserved 2012
R. Sabatowski ${ }^{1,2} \cdot$ U. Kaiser ${ }^{1}$

${ }^{1}$ UniversitätsSchmerzCentrum, Universitätsklinikum Carl Gustav Carus, Technische Universität Dresden

${ }^{2}$ Klinik und Poliklinik für Anaesthesiologie und Intensivtherapie, Universitätsklinikum Carl Gustav Carus, Technische Universität Dresden

\section{Multimodale Schmerztherapie}

\section{Ein etabliertes Verfahren?}

Chronische Schmerzen sind ein häufiges Phänomen mit erheblichen psychosozialen und auch gesamtgesellschaftlich-ökonomischen Konsequenzen. Eine Datenerhebung für Europa ergab für Deutschland, dass etwa 11 Mio. Menschen unter chronischen Schmerzen leiden [3]. Meist bestanden die Schmerzen schon länger, bei jedem fünften Patienten mehr als 20 Jahre. Zu den häufigsten Schmerzarten zählten Schmerzen des muskuloskeletalen Systems inkl. chronischer Rückenschmerzen. Nur 2\% der betroffenen Patienten wurden von Ärzten betreut, die sich auf dem Gebiet der Schmerztherapie spezialisiert hatten. Die Schmerzen führten beim überwiegenden Teil der befragten Patienten zu erheblichen Einschränkungen der Aktivitäten des täglichen Lebens, teilweise mit Auswirkungen auf die Arbeitsfähigkeit [3].

Diese Zahlen aus dem europäischen Survey wurden im Wesentlichen von Wolff et al. [21] durch eine Datenbankanalyse bestätigt. Die Prävalenz lag in dieser Untersuchung nur geringfügig höher und wurde mit einem Anteil von $17 \%$ der deutschen Gesamtbevölkerung angegeben, wobei Rückenschmerzen besonders häufig vorkamen. Auffällig war hier, dass die eingesetzten Therapien überwiegend passiver Natur waren. $\mathrm{Zu}$ den am häufigsten eingesetzten Verfahren zählten orale Medikamente (54\% der Patienten), Wärmebehandlungen (30\%), Massage (53\%) und Schlammpackungen (32\%). Insgesamt wurden die bestehen- den Behandlungen bzw. Behandlungsstrategien als unzureichend klassifiziert [21].

\section{„Risikofaktor“ Kosten}

Neben der medizinischen Behandlung sind auch ökonomische Aspekte in der Therapie chronischer Schmerzpatienten relevant. Am Beispiel von chronischen Rückenschmerzen konnte gezeigt werden, dass die durchschnittlichen Kosten pro Patient und Jahr in Deutschland bei etwa $1300 €$ liegen. Dabei beträgt der Anteil der sog. indirekten Kosten, zu denen etwa der schmerzbedingte Produktionsausfall zählt, 54\% der Gesamtkosten. Mit steigendem Chronifizierungsstadium können sich die direkten und indirekten Kosten vervielfachen [20]. Auch führen Faktoren, die die Erkrankung komplizieren, z. B. eine neuropathische Schmerzkomponente beim chronischen Rückenschmerz oder komorbide psychische Erkrankungen, zu einem disproportionalen Anstieg der Gesamtkosten [2, 18]. Allein diese Zahlen verdeutlichen, dass in Deutschland ein hoher Bedarf an schmerztherapeutischen Einrichtungen und Therapieprogrammen besteht.

Monokausale Therapieansätze, wie aus dem europäischen Schmerzsurvey von Breivik et al. [3] sowie der Datenanalyse von Wolff et al. [21] ersichtlich, scheinen zu überwiegen, ohne dass aber deren Effektivität und der daraus zu erwartende Nutzen für viele Patienten nachgewie- sen wurde. Niemier [14] konnte in einer aktuellen Untersuchung zeigen, dass etwa wiederholte „stationäre Komplexbehandlungen " mit Schwerpunkt auf interventionellen Verfahren, z. B. periradikuläre Injektionen, zu keiner Verbesserung der Erkrankung führten. Vielmehr stiegen die Anzahl der schmerzhaften Regionen sowie der Analgetikabedarf und es wurde eine negative psychosoziale Entwicklung im Behandlungsverlauf beobachtet. Dagegen wurde die Effektivität multimodaler integrativer Therapieprogramme bereits vielfach in internationalen und nationalen Studien belegt $[6,7$, $10,15,19]$. In der aktuellen VersorgungsLeitlinie Kreuzschmerz wird dieser Ansatz mit hohem positivem Empfehlungsgrad aufgeführt [4]. Dabei zeigten sich die Effekte der multimodalen Therapie nicht nur in unterschiedlichen Items verschiedener standardisierter Schmerzfragebögen, sondern es konnte auch deren Kosteneffektivität nachgewiesen werden. Nagel u. Korb [12] erzielten bei berufstätigen Rückenschmerzpatienten einen Rückgang der Arbeitsunfähigkeitstage um etwa $75 \%$ und eine Halbierung der schmerzbedingten Behandlungen bzw. Arzttermine bei gleichzeitig deutlichen Effekten im Sinne einer Verbesserung der Lebensqualität und anderer Parameter. Dies führte bereits nach einem Jahr zu einer Einsparung der schmerzbedingten Kosten von 2000-6000 € für Patienten mit anfänglich höherer Beeinträchtigung. 


\section{Was bedeutet „multimodale Schmerztherapie"?}

Die multimodale integrative Schmerztherapie wurde von der Ad-hoc-Kommission „Multimodale interdisziplinäre Schmerztherapie“ der Deutschen Schmerzgesellschaft als „gleichzeitige, inhaltlich, zeitlich und in der Vorgehensweise aufeinander abgestimmte umfassende Behandlung von Patienten mit chronifizierten Schmerzsyndromen bezeichnet, in die verschiedene somatische, körperlich übende, psychologisch übende und psychotherapeutische Verfahren nach vorgegebenem Behandlungsplan mit identischem, unter den Therapeuten abgesprochenem Therapieziel eingebunden sind“ [1].

Dieses teamorientierte Vorgehen bedarf einer optimierten Organisationsstruktur. Im Sinne des Prozessmanagements lassen sich multimodale integrative Therapiemodelle als sog. horizontal ausgerichtete Gesamtprozesse darstellen. Das bedeutet, dass klassisch orientierte Organisationsformen, in denen verschiedene Behandlungsstrukturen parallel und nur mit gering ausgeprägter Kommunikation untereinander agieren, zugunsten einer engen zeitlichen, inhaltlichen sowie sektorenübergreifenden Ausrichtung aufgegeben werden [5]. Zur Basis der Implementierung optimierter Prozessabläufe in der Schmerztherapie zählen u. a. standardisierte und überprüfbare Struktur- sowie Prozessqualitätsmerkmale [17]. Diese wurden für die multimodale Schmerztherapie im Jahr 2009 in einem ersten Schritt definiert [1]. Betrachtet man die gegenwärtige Versorgungssituation der Schmerzmedizin in Deutschland, so fällt auf, dass eine Vielzahl von Einrichtungen unter dem Label „multimodale Schmerztherapie“ auftreten, ohne dass die geforderten Qualitätsmerkmale erkennbar erfüllt werden.

Ein Grund hierfür könnte darin bestehen, dass diese Qualitätsparameter nicht umsetzbar sind. Nagel et al. [13] zeigten jedoch in ihrer aktuellen Untersuchung anhand einer ausgewählten Stichprobe, dass die Qualitätsmerkmale durchaus zu erzielen sind. So wird z. B. von allen teilnehmenden Einrichtungen vor dem eigentlichen Therapieprogramm ein interdisziplinäres Assessment durchge- führt. Dies ist umso erfreulicher, als Rothman et al. [16] in einer Untersuchung an Patienten mit chronischen bewegungsassoziierten Schmerzen nachweisen konnten, dass allein die Durchführung eines multimodalen Assessments vor der Behandlung zu einem positiven und statistisch signifikanten Therapieeffekt hinsichtlich verschiedener Lebensqualitätsparameter und einer höheren Patientenzufriedenheit führte. Auch andere Strukturparameter, wie Raumstruktur, Gruppengrößen, Durchführung von regelmäßigen Teamsitzungen, Prozessparameter sowie der Anteil körperlich aktivierender Therapiebausteine, konnten in der weit überwiegenden Zahl der Einrichtungen in der Untersuchung von Nagel et al. [13] nachgewiesen werden. Dabei ist noch auf 2 Punkte besonders hinzuweisen: Es handelte sich bei den teilnehmenden Einrichtungen nicht nur um große Kliniken mit möglicherweise größeren Ressourcen, sondern teilweise auch um Einrichtungen, die sich in „kleineren“ Krankenhäusern in ländlicher Region etablieren konnten.

\section{Mehr Bedeutung für das Teamwork}

Ein weiterer Punkt, der zu beachten ist, betrifft die Qualifikation der Mitarbeiter. Alle multimodalen Programme wurden von Ärzten mit schmerztherapeutischer Zusatzqualifikation geleitet. Darüber hinaus wiesen aber bereits 56\% der Einrichtungen die Zusatzqualifikation „spezielle Schmerzpsychotherapie" für ihre Mitarbeiter nach, obwohl diese Zusatzqualifikation erst seit kurzer Zeit zu erlangen und bisher auch nicht in vollem Umfang in der Schmerzmedizin etabliert und implementiert ist [13].

Nagel et al. [13] demonstrieren, dass das Attribut „multimodal“ durchaus im Sinne eines horizontalen Gesamtprozesses umsetzbar ist, und wenden sich zu Recht gegen eine inflationäre Nutzung dieses Begriffs, ohne dass entsprechende Merkmale hinterlegt sind. Dies ist auch im Kontext einer Diskussion um horizontale und vertikale Prozesse, wie sie aktuell wieder geführt wird, von großer Bedeutung. In dieser Kontroverse wird die Sinnhaftigkeit einer koordinierten Diagnostik und Betreuung durch ein mul- tiprofessionelles Team zugunsten eines „umfassend ausgebildeten Schmerzmediziners ..., der die schmerzmedizinische Kompetenz verschiedener Fachgebiete in sich vereint“ in Frage gestellt [11]. Dieser Schmerzmediziner solle „im Sinne eines Querschnittfaches ausgebildet“ sein [11]. Hier muss noch vor der Frage der Umsetzbarkeit (welche Qualifikationen sind beispielsweise erforderlich?) die Frage der Sinnhaftigkeit gestellt werden. Auch eine umfassende Ausbildung kann die in der multimodalen Therapie erforderliche interdisziplinäre Diskussion und v. a. den teamorientierten Therapieansatz nicht ersetzen.

Natürlich sind in der multimodalen integrativen Schmerztherapie noch viele wichtige Fragen unbeantwortet. So bedarf es der Etablierung und Anerkennung des multimodalen Assessments als eigenständiger Baustein. Darüber hinaus müssen die Kernprozesse, u. a. medizinische, psychologische und physiotherapeutische Therapieverfahren, noch definiert und evaluiert werden. Outcomeparameter sind $\mathrm{zu}$ definieren, beschreiben und untersuchen. Gerade diese scheinen von vielen Faktoren abhängig zu sein. Einen wichtigen Aspekt dabei stellt auf Seiten der Patienten die sog. Veränderungsmotivation dar. Die multimodale Schmerztherapie kann nur dann zu einer positiven Veränderung des Erlebens und Verhaltens führen, wenn bei den Patienten eine Bereitschaft zur Umsetzung eines selbstständigen aktiven Schmerzmanagements vorhanden ist. Küchler et al. [9] konnten anhand einer Stichprobe von 169 Patienten zeigen, dass es im Rahmen eines standardisierten multimodalen Therapieprogramms zu einer Änderung der Veränderungsmotivation zugunsten der Phase der Handlung, d. h. des aktiven strukturierten Verhaltens, sowie der Phase der Aufrechterhaltung, d. h. der Anwendung neuer Strategien und erlernter Fertigkeiten über einen längeren Zeitraum hinweg, kam. Auch konnten die Autoren zeigen, dass es nach einer Wiederholungswoche, wie sie in vielen Einrichtungen mittlerweile zum Standard gehört, zu einer Stabilisierung der Motivationsänderung gekommen war. Neben diesen durchaus positiven Effekten wiesen sie aber auch darauf hin, dass bei differenzierter Betrachtung 
Patientengruppen identifizierbar waren, bei denen keine Änderung der Motivation beobachtet werden konnte [9]. Dieser Aspekt führt neben der notwendigen Diskussion um Kernprozesse und Effektivitätsparameter der multimodalen Therapie auch zwangsläufig dazu, in Zukunft die Patientenpopulationen differenzierter zu betrachten. Nicht nur die Therapie ist komplex, auch die Patienten mit ihrem individuellen soziodemographischen und kulturell-lernbedingten Hintergrund sowie unterschiedlichen Krankheitsausmaß (z. B. Anzahl und Art komorbider Störungen) sind es.

Eine Unterscheidung in bestimmte Patientengruppen hinsichtlich patientenimmanenter Charakteristika und Verlaufsbesonderheiten wird helfen, die gewünschten Therapieeffekte besser abzubilden, aber v. a. auch die Kernprozesse, d. h. die eigentlichen Therapiebausteine, im Sinne einer subgruppenspezifischeren Behandlung auszurichten und sowohl die Passung zwischen Patient und Angebot als auch die Passung an den Schnittstellen der Zuweisung zu bestimmten Angeboten zu optimieren [8].

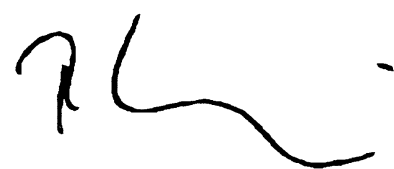

R. Sabatowski

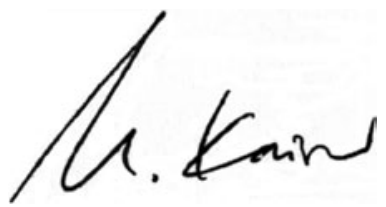

U. Kaiser

\section{Korrespondenzadresse}

Prof. Dr. R. Sabatowski

UniversitätsSchmerzCentrum, Universitätsklinikum Carl Gustav Carus,

Technische Universität Dresden

Fetscherstr. 74, 01307 Dresden

rainer.sabatowski@uniklinikum-dresden.de

\section{Literatur}

1. Arnold B, Brinkschmidt T, Casser HR et al (2009) Multimodale Schmerztherapie. Konzepte und Indikation. Schmerz 23:112-120
2. Baumeister $H$, Knecht $A$, Hutter N (2012) Direct and indirect costs in persons with chronic back pain and comorbid mental disorders - a systematic review. J Psychosom Res 73:79-85

3. Breivik H, Collett B, Ventafridda V et al (2006) Survey of chronic pain in Europe: prevalence, impact on daily life, and treatment. Eur J Pain 10:287-333

4. Bundesärztekammer (BÄK), Kassenärztliche Bundesvereinigung (KBV), Arbeitsgemeinschaft der Wissenschaftlichen Medizinischen Fachgesellschaften (AWMF) (2011) Nationale VersorgungsLeitlinie Kreuzschmerz - Langfassung. Version 1.2.2011. http://www.versorgungsleitlinien.de/ themen/kreuzschmerz. Zugegriffen: 2 . November 2012

5. Dunkel M, Kramp M (2012) Multimodale Schmerztherapie. Implementierung eines Prozessmanagements. Anästhesiol Intensivmed Notfallmed Schmerzther 47:504-510

6. Guzman J, Esmail R, Karjalainen K et al (2001) Multidisciplinary rehabilitation for chronic low back pain: systematic review. BMJ 322:1511-1516

7. Jensen IB, Bergström $G$, Ljungquist T, Bodin $L$ (2005). A 3-year follow-up of a multidisciplinary rehabilitation program for back and neck pain. Pain 115:273-283

8. Kaiser U, Schütze A, Balck F, Sabatowski R (2012) Auf der Suche nach Subgruppen für den Therapieverlauf - erste Analysen zur Responderidentifikation Fast Forward. Schmerz 26 (Suppl 1):124

9. Küchler A, Sabatowski R, Kaiser U (2012) Veränderungsmotivation bei Patienten mit chronischer Schmerzerkrankung nach einer multidisziplinären Behandlung. Schmerz 26:670-676

10. Mattenklodt $P$, Ingenhorst A, Wille $C$ et al (2008) Multimodale Gruppentherapie bei Senioren mit chronischen Schmerzen. Schmerz 22:551-561

11. Müller-Schwefe GHH (2012) Wer ... Schmerzmedizin 28:2-3

12. Nagel B, Korb J (2009) Multimodale Therapie des Rückenschmerzes. Orthopade 38:907-912

13. Nagel B, Pfingsten M, Brinkschmidt T et al (2012) Struktur- und Prozessqualität multimodaler Schmerztherapie. Schmerz 26:661-669

14. Niemier K (2012) Langzeiteffekte interventioneller Behandlungen von chronischen Schmerzen des Bewegungssystems. Schmerz 26:185-191

15. Pöhlmann K, Tonhauser T, Joraschky P, Arnold B (2008) Die multimodale Schmerztherapie Dachau (MSD). Daten zur Wirksamkeit eines diagnose-unabhängigen multimodalen Therapieprogramms bei Rückenschmerzen und anderen Schmerzen. Schmerz 23:40-46

16. Rothman MG, Ortendahl M, Rosenblad A, Johansson AC (2012) Improved quality of life, working ability, and patient satisfaction after a pretreatment multimodal assessment method in patients with mixed chronic muscular pain: a randomized-controlled study. Clin J Pain (im Druck)

17. Sabatowski R, Maier C, Willweber-Strumpf $A$ et al (2011) Empfehlung zur Klassifikation schmerztherapeutischer Einrichtungen in Deutschland. Schmerz 25:368-376

18. Schmidt CO, Schweikert B, Wenig CM et al (2009) Modelling the prevalence and cost of back pain with neuropathic components in the general population. Eur J Pain 13:1030-1035

19. Schütze A, Kaiser U, Ettrich U et al (2009) Evaluation einer multimodalen Schmerztherapie am UniversitätsSchmerzCentrum Dresden. Schmerz 23:609-617

20. Wenig CM, Schmidt CO, Kohlmann T, Schweikert B (2009) Costs of back pain in Germany. Eur J Pain 13:280-286

21. Wolff R, Clar C, Lerch C, Kleijnen J (2011) Epidemiologie von nicht tumorbedingten chronischen Schmerzen in Deutschland. Schmerz 25:26-44

\section{Antirheumatika bremsen Fortschreiten von Morbus Bechterew}

Nicht-steroidale Antirheumatika (NSAR) lindern die Schmerzen bei chronischen Entzündungen wie Morbus Bechterew. Aktuelle Studien zeigen, dass eine regelmäBige NSAR-Einnahme über einen längeren Zeitraum nicht nur die Symptome lindern, sondern auch das Fortschreiten der Krankheit bremsen. In der Studie wurden 88 Patienten über zwei Jahre beobachtet. Die Versteifung der Wirbelsäule verschlimmerte sich bei den Patienten, die NSAR seltener oder in kleineren Dosen als empfohlen eingenommen hatten. Bei Patienten die mindestens $50 \%$ der maximalen empfohlenen Dosis täglich einnahmen, fiel keine weitere Verknöcherung der Wirbelsäule auf. Von der Behandlung profitieren vor allem Patienten, die das höchste Risiko für eine Verschlimmerung der Krankheit haben. Dies sind Patienten mit hohen Werten des Entzündungsmarkers C-reaktives Protein (CRP) oder mit Syndesmophyten.

Quelle: Deutsche Gesellschaft für Rheumatologie e.V. www.dgrh.de

Literatur:

Poddubnyy D, Rudwaleit M, Haibel $\mathrm{H}$ et al (2012) Effect of non-steroidal anti-inflammatory drugs on radiographic spinal progression in patients with axial spondyloarthritis: results from the German Spondyloarthritis Inception Cohort. Annals of Rheumatic Diseases, 71:1616-1622 